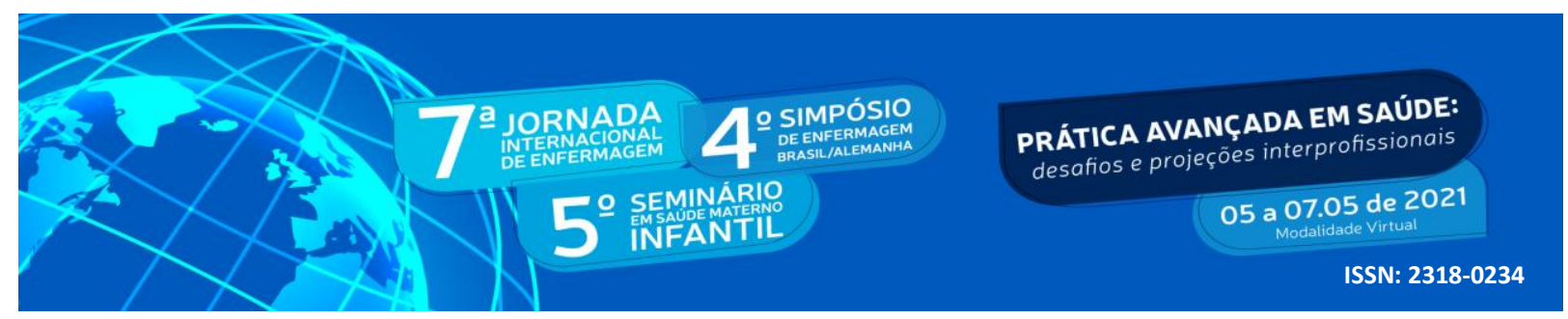

DOI: http://doi.org/10.48195/jie2021-126

\title{
CAPACIDADE DE TOMADA DE DECISÃO E SUA RELAÇÃO COM O COMPORTAMENTO ALIMENTAR NA INFÂNCIA
}

\author{
Michele Vargas Reginatto ${ }^{1}$; Ana Paula Gularte Barbosa² ${ }^{2}$ Marcia Montagner ${ }^{3}$; \\ Franceliane Jobim Benedetti ${ }^{4}$; Josiane Lieberknecht Wathier Abaid ${ }^{5}$
}

\begin{abstract}
RESUMO
O objetivo da pesquisa foi identificar, na literatura, abordagens sobre os fatores que influenciam a capacidade de tomada de decisão e sua relação com o comportamento alimentar na infância frente às escolhas alimentares. Realizou-se uma revisão narrativa nos meses de março a junho de 2018, em periódicos da área da saúde. Encontrou-se $42,9 \%$ estudos qualitativos, os que mais trataram do tema e $57,1 \%$ quali e quantitativos; entrevistas e questionários foram os mais frequentemente utilizados como ferramentas na investigação do comportamento alimentar, escolhas e preferências e a intervenção como forma de abordagem positiva para influenciar a capacidade de tomada de decisão para escolhas saudáveis de alimentos. A tomada de decisão é uma função executiva complexa, desenvolvida ao longo da idade escolar e, após uma intervenção e oferta de alimentos saudáveis e não saudáveis ao mesmo tempo, podem influenciar na capacidade de tomada de decisão para escolhas de alimentos saudáveis.
\end{abstract}

Palavras-chave: Tomada de Decisão; Comportamento Alimentar; Crianças.

\begin{abstract}
The objective of the research was to identify, in the literature, approaches on the factors that influence the decision-making ability and its relationship with eating behavior in childhood in the face of food choices. A narrative review was carried out from March to June 2018, in health journals. 42.9\% qualitative studies were found, the ones that most dealt with the theme and $57.1 \%$ quali and quantitative studies; interviews and questionnaires were the most frequently used as tools in the investigation of eating behavior, choices and preferences and intervention as a form of positive approach to influence the decision-making ability for healthy food choices. Decision making is a complex executive function, developed throughout school age and, after an intervention and offering healthy and unhealthy foods at the same time, can influence the decision-making ability for healthy food choices.
\end{abstract}

Keywords: Decision making; Feeding Behavior; Children.

\footnotetext{
${ }^{1}$ Ms. em Saúde Materno Infantil. Universidade Franciscana - UFN. E-mail: mi.reginatto@ gmail.com

${ }^{2}$ Ms. em Saúde Materno Infantil. Universidade Franciscana - UFN. E-mail: anapaulab6@gmail.com

${ }^{3}$ Ms. em Saúde Materno Infantil. Universidade Franciscana - UFN. E-mail:marcia.montagner@ufn..edu.br

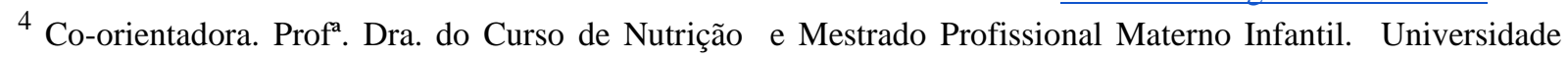
Franciscana - UFN. E-mail: franceliane@ufn.edu.br

5 Orientadora. Prof ${ }^{a}$. Dra. do Curso de Psicologia e Mestrado Profissional Materno Infantil. Universidade Franciscana - UFN. E-mail: josianelieb@ufn.edu.br
} 


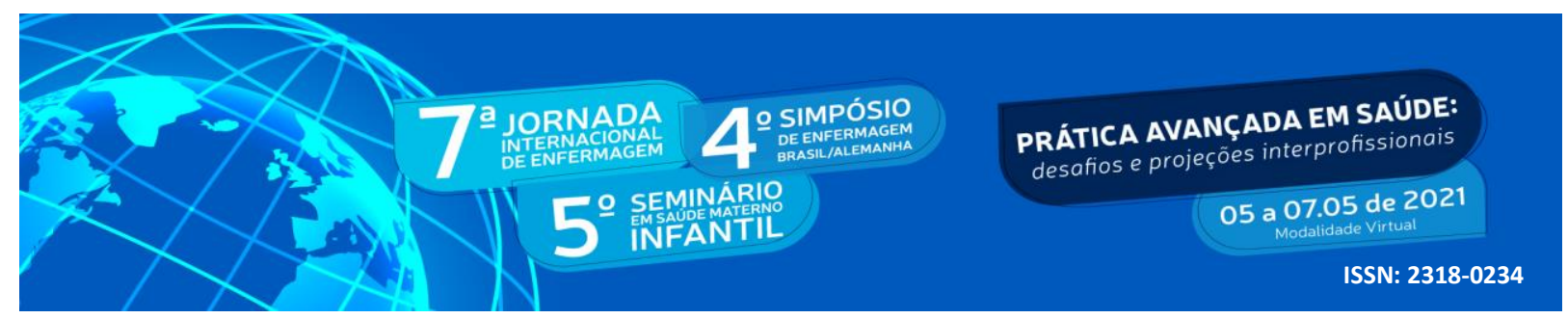

\section{INTRODUÇÃO}

$\mathrm{O}$ ato de selecionar e ingerir alimentos preferidos é considerado um comportamento aprendido, que evolui desde os primeiros dias, influenciado pela maturidade, ambiente de socialização, pelos fatores afetivos e pela interação criança-família. A alimentação infantil é determinada pelas preferências da criança, que manifestam-se, geralmente, em torno dos alimentos ricos em lipídios e doces. E, esta preferência, provavelmente inata, declina se a criança tiver pouca oportunidade de consumir esses alimentos. As crianças tendem a rejeitar os alimentos que provam pela primeira vez, mas ao repeti-los em outras oportunidades, passam a aceitá-los (VIANA; SANTOS; GUIMARÃES, 2008).

Durante a infância, o ato de alimentar-se é, inicialmente, guiado por um estado de depleção de nutrientes, mas evidências indicam que ,mesmo durante os primeiros anos de vida, os controles de ingestão alimentar tornam-se mais complexos, como quando aprende-se a comer em resposta à presença de alimentos palatáveis, às configurações sociais, ao estado emocional, bem como atitudes, conhecimento e crenças quanto aos alimentos saudáveis (BIRCH, 1998).

A influência social tem mostrado moldar as preferências alimentares das crianças. Modelar o comportamento alimentar positivo é, portanto, importante para que as crianças imitem esse comportamento. Desta forma, a influência dos pais sobre a ingestão alimentar de seus filhos está mais relacionada ao seu próprio comportamento alimentar do que à forma como eles podem insistir em certos alimentos sendo ingeridos (DECOSTA et al., 2017).

É na fase escolar, a partir dos seis anos, que as crianças começam a ter mais liberdade e socializam mais, seja na rua, escola, shopping ou supermercados. A oferta de alimentos diversos, coloridos e saborosos, nestes locais, normalmente não precisam ser apresentados às crianças, pois elas já tiveram acesso a eles através da agressiva propaganda das indústrias alimentícias (QUAIOTI; ALMEIDA, 2006).

\section{OBJETIVO}

Identificar na literatura científica as abordagens sobre os fatores que influenciam a capacidade de tomada de decisão e sua relação com o comportamento alimentar na infância frente às escolhas alimentares. 


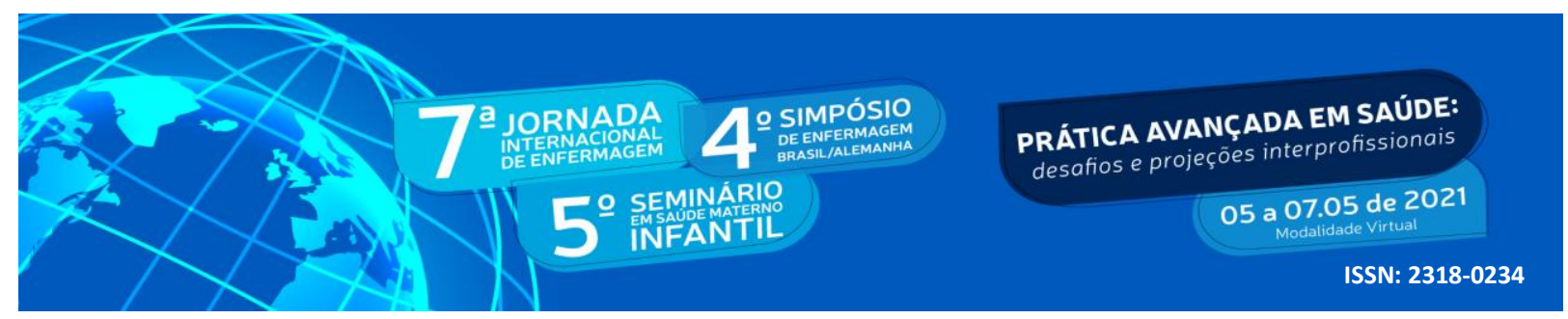

\section{METODOLOGIA}

Realizou-se uma revisão narrativa da literatura, nos meses de março a junho de 2018, em base de dados MEDLINE, National Library of Medicine (PubMed), Biblioteca Virtual em Saúde - BVS e PsycINFO - APA), com o propósito de identificar as abordagens sobre os fatores que influenciam a capacidade de tomada de decisão e sua relação com o comportamento alimentar na infância frente às escolhas alimentares.

Utilizou-se os seguintes descritores "tomada de decisões", "comportamento alimentar", "nutrição da criança", "comportamento infantill”; os descritores MeSH: "decision making", "feeding behavior", "child nutrition", "child behavior"; e os descritores THESAURUS: "decision making", "eating behavior", "child care", "nutrition" e o operador booleano "AND" entre os termos.

Como critérios de inclusão: sem limitação temporal de publicação; artigos cujas pesquisas tenham sido realizadas com indivíduos em desenvolvimento típico; faixa etária entre 6 e 9 anos; artigos que tivessem aplicado alguma ferramenta investigativa (questionário, entrevista, teste, entre outros) com crianças e estudos disponibilizados na íntegra. Já os critérios de exclusão: monografias; dissertações; teses; revisões; apresentações em eventos; os artigos que abordavam indivíduos enfermos com padrão alimentar em condições específicas de saúde (HIV-positivo, diabetes, hipertensão, entre outras) e faixa etária inferior a seis e superior a 10 anos.

\section{RESULTADOS E DISCUSSÃO}

Percebeu-se que os artigos produzidos no período de 2006 a 2017, na sua maioria foram produzidos nos Estados Unidos (57,1\%); seguidos pela Inglaterra $(28,6 \%)$ e Havaí $(14,3 \%)$ e destes, $(71,4 \%)$ dispostos na base de dados PubMed.

Verificou-se que as metodologias usadas dividiram-se entre qualitativas (42,9\%), as mais utilizados para tratar do tema e qualitativas e quantitativas $(57,1 \%)$ e as ferramentas, para a investigar o comportamento alimentar, escolhas e preferências utilizadas para coleta de dados são, normalmente, entrevistas e questionários. 


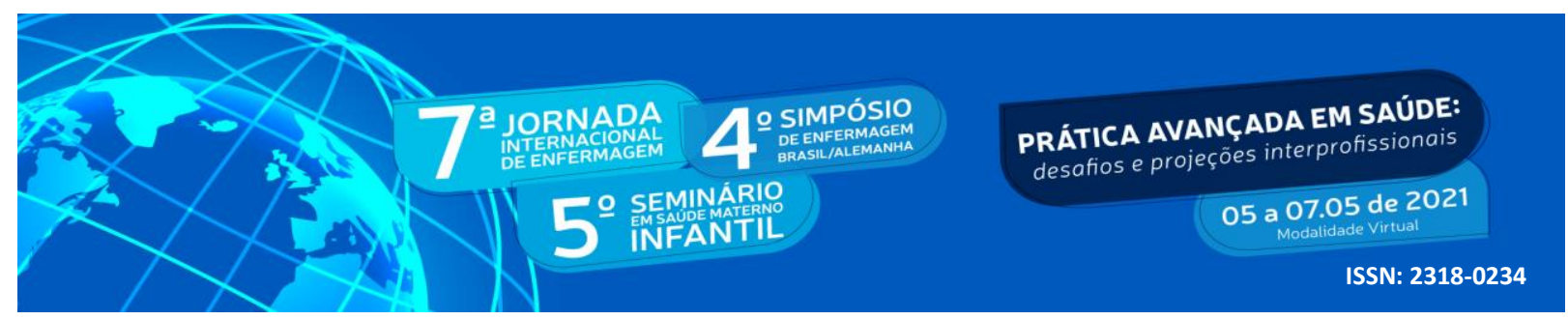

Quanto ao conteúdo, os artigos identificaram uma maior capacidade de tomada de decisão para escolhas saudáveis de alimentos, normalmente após uma intervenção. Em Smith e Cunningham-Sabo (2014), as intervenções comportamentais efetivas, combinadas com marketing, comunicação e economia comportamental, provavelmente, são necessárias para encorajar maior consumo de vegetais para atender aos novos padrões alimentares. Gittelsohn et al. (2010), realizaram intervenção em um período de 9 a 11 meses, em cinco lojas de alimentos no Havaí, como impactos positivos, por parte das crianças, maior ingestão de água e de alimentos integrais .

Heard et al. (2016), também demonstram a importância de desenvolver intervenções que impactem positivamente as preferências das crianças por alimentos saudáveis. Korinek et al (2015), constatou que crianças mais expostas a alguns tipos de frutas e verduras (FV) em casa, as consomem mais na escola e tendem a selecionar preparações saudáveis, inclusive, aquelas não tão familiares, quando oferecidas no ambiente escolar.

Nota-se uma crescente participação das crianças na hora da compra dos alimentos, resultado do marketing ativo voltado a este público, via televisão e outras mídias e, é considerada, pela maioria dos pais, como uma influência importante (CAMPBELL; CRAWFORD; HESKETH, 2006). No entanto, O’Dougherty, Story e Stang (2006), ressaltam que os pais podem ser um elo "forte" ou "fraco" no processo de seleção de alimentos para crianças, indo ao encontro de estudos, que enfatizam o papel dos pais na modulação das preferências alimentares das crianças, principalmente, por serem a primeira fonte de socialização do indivíduo (BOGL et al., 2017; SALVY et al., 2011) e, ainda, os pais encorajam as crianças a desenvolverem critérios para seleção de alimentos, durante as compras no supermercado, embora tanto para doces e lanches quanto para frutas e vegetais.

Pinard et al.(2014), referem que os pais expressam preocupação com as influências de marketing sobre as preferências alimentares e o consumo de seus filhos. À medida que as crianças amadurecem, tornam-se consumidores independentes, tornando a exposição à comercialização e o desenvolvimento de preferências de gosto muito importantes nos primeiros anos. A importância da capacidade de tomada de decisão frente às escolhas alimentares na infância pode prevenir desfechos como sobrepeso e obesidade e o ato de comer refeições fora de casa pode estar associado a estes desfechos, além de proporcionar um consumo maior de alimentos não saudáveis (KASPARIAN et al., 2017). 


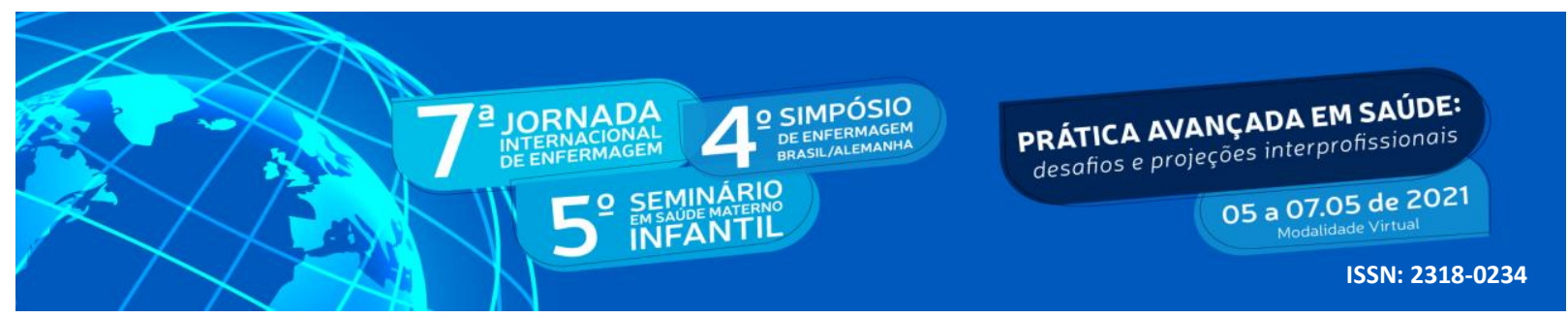

Pinard et al (2014), discutem que a tomada de decisão entre díades de pais e filhos sobre as escolhas alimentares em restaurantes é complexa, pois há a influência ao nível individual, como as preferências de gosto e ao nível ambiental, como o marketing de alimentos. Desta forma, o marketing positivo a favor de uma alimentação saudável pode promover uma mudança nos padrões alimentares. Em Sharps e Robinson (2016), crianças expostas a mensagens sobre os benefícios para a saúde de comer frutas e verduras, aumentaram, significativamente, o seu consumo.

Outro fator que impacta a capacidade de tomada de decisão, principalmente, na infância, é a oferta de alimentos saudáveis e não saudáveis ao mesmo tempo. Em Beets et al. (2014), crianças com a oportunidade da escolha entre frutas ou lanches açucarados, salgadinhos ou lanches integrais, quase nunca selecionam frutas. Estes resultados indicam que quando as políticas recomendam servir frutas como um lanche, sem explicitar, que lanches alternativos não podem ser oferecidos simultaneamente, é improvável que eles tenham o efeito desejado, que é de promover uma alimentação mais saudável (BRASIL, 2010).

Há um reflexo preciso dos padrões de consumo das crianças quando expostas a diferentes escolhas de lanches. Marty et al. (2017), ao abordarem atitudes hedônicas frente às escolhas alimentares, identificaram que as crianças escolhiam, principalmente, os alimentos de que gostavam, independentemente de suas atitudes. Quando suas pontuações hedônicas implícitas e explícitas diminuíram, as crianças escolheram os alimentos que percebiam como menos saudáveis, mesmo que gostassem dos bufês saudáveis e não saudáveis de forma igualitária. Isso demonstra que variedade, moderação e proporcionalidade são conceitos que precisam ser introduzidos como estratégias que auxiliem a tomada de decisões sem altos níveis de processamento cognitivo (FRERICHS et al., 2016).

\section{CONCLUSÃO}

Conclui-se, que a tomada de decisão é uma função executiva complexa e que é desenvolvida ao longo da idade escolar e observou-se nos artigos, que após uma intervenção e oferta de alimentos saudáveis e não saudáveis ao mesmo tempo, podem influenciar na capacidade de tomada de decisão para escolhas de alimentos saudáveis. Reforça-se, portanto, a necessidade de novos estudos para a faixa etária escolar e que abordem a relação "tomada de 


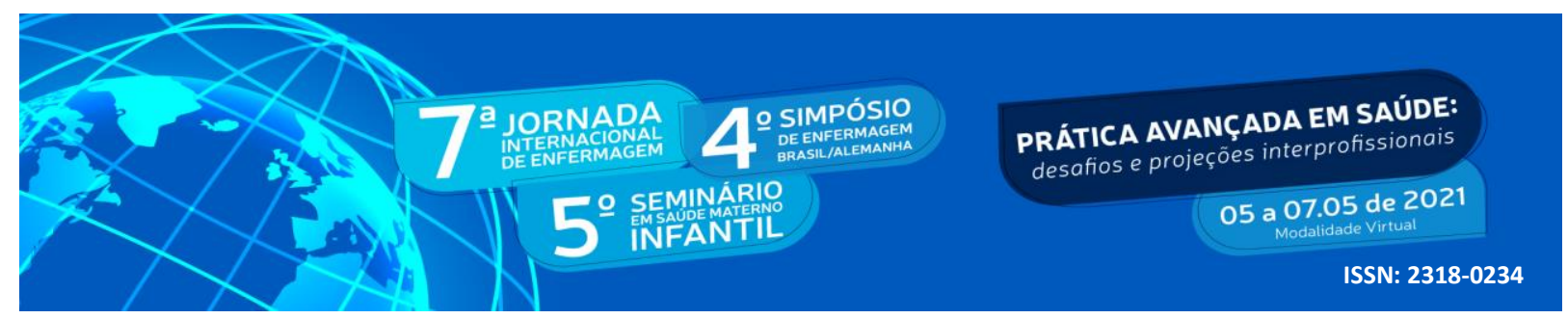

decisão" e "comportamento alimentar".

\section{REFERÊNCIAS}

BEETS, Michael W. et al. Children select unhealthy choices when given a choice among snack offerings. Journal of the Academy of Nutrition and Dietetics, v. 114, n. 9, p. 14401446, 2014.

BIRCH, L. L. Symposium: The Effects of Childhood Diet on Adult Health and Disease Psychological Influences on the Childhood Diet 1. The Journal of Nutrition, p. 407-410, 1998.

BOGL, L. H. et al. Familial resemblance in dietary intakes of children, adolescents, and parents: Does dietary quality play a role? Nutrients, v. 9, n. 892, 2017.

BRASIL. Manual das cantinas escolares saudáveis: promovendo a alimentação saudável. 2010 .

CAMPBELL, K. J.; CRAWFORD, D. A.; HESKETH, K. D. Australian parents' views on their 5-6-year-old children's food choices.Health Promotion InternationalCampbell, Karen J.: Centre for Physical Activity and Nutrition Research, School of Exercise and Nutrition Sciences, Deakin University, 221 Burwood Highway, Burwood, VIC, Australia, 3125, kcamp@deakin.edu.auOxford University Press, , 2006.

DECOSTA, P. et al. Changing children's eating behaviour - A review of experimental research. Appetite, v. 113, p. 327-357, jun. 2017.

DIAS, N. M.; SEABRA, A. G. Funções Executivas : Desenvolvimento E Intervenção Funções Executivas : Desenvolvimento E Intervenção. Temas sobre Desenvolvimento, v. 19, n. 107, p. 206-212, 2013.

\section{FINEOUT-OVERHOLT, E.; MELNYK, B. Evidence-based practice in nursing \&}

healthcare: A guide to best practice. [s.l: s.n.]. v. 19

FRERICHS, L. et al. Children's Discourse of Liked, Healthy, and Unhealthy Foods. Journal of the Academy of Nutrition and Dietetics, v. 116, n. 8, p. 1323-1331, ago. 2016.

GITTELSOHN, Joel et al. A food store intervention trial improves caregiver psychosocial factors and children's dietary intake in Hawaii. Obesity, v. 18, n. S1, p. S84-S90, 2010.

HEARD, A. M. et al. Piloting an online grocery store simulation to assess children's food choices. Appetite, v. 96, p. 260-267, jan. 2016.

KASPARIAN, M. et al. Parenting practices toward food and children's behavior: Eating away from home versus at home. Appetite, v. 114, p. 194-199, jul. 2017.

KORINEK, Elizabeth V. et al. Fruit and vegetable exposure in children is linked to the selection of a wider variety of healthy foods at school. Maternal \& child nutrition, v. 11, n. 4, p. 999-1010, 2015. 


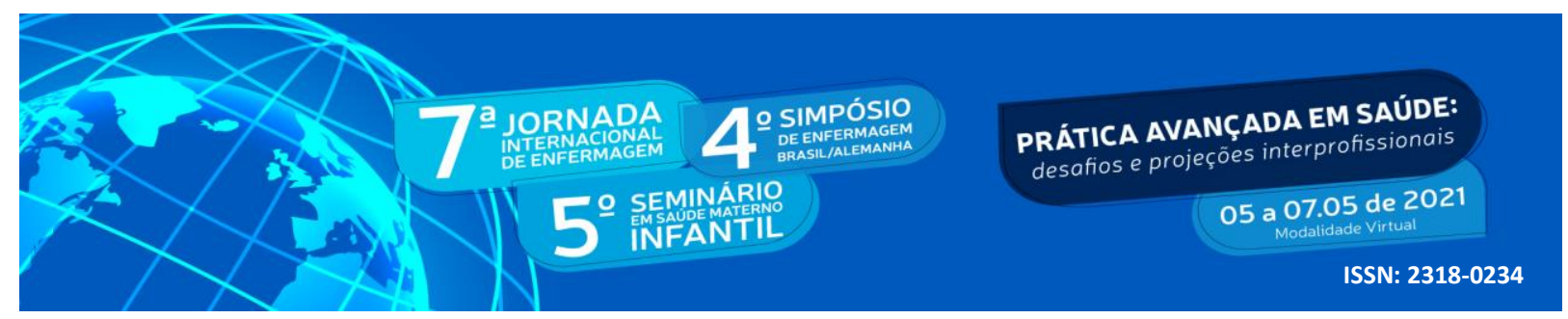

MARTY, Lucile et al. Do hedonic-versus nutrition-based attitudes toward food predict food choices? A cross-sectional study of 6-to 11-year-olds. International Journal of Behavioral Nutrition and Physical Activity, v. 14, n. 1, p. 1-10, 2017.

O’DOUGHERTY, Maureen; STORY, Mary; STANG, Jamie. Observations of parent-child co-shoppers in supermarkets: children's involvement in food selections, parental yielding, and refusal strategies. Journal of nutrition education and behavior, v. 38, n. 3, p. 183-188, 2006.

PINARD, Courtney A. et al. Influences on food away from home feeding practices among English and Spanish speaking parent-child dyads. Journal of Child and Family Studies, v. 24, n. 7, p. 2099-2106, 2015.

QUAIOTI, T. C. B.; ALMEIDA, S. DE S. Determinantes Psicobiológicos Do Comportamento Alimentar: Uma Ênfase em Fatores Ambientais que Contribuem Para a Obesidade. Universidade do Sagrado Coração - USC Universidade de São Paulo - USP, v. 17, n. 4, p. 193-211, 2006.

SALVY, S.-J. et al. Influence of parents and friends on children's and adolescents' food intake and food selection. Am J Clin Nutr, v. 93, p. 87-92, 2011.

SHARPS, Maxine; ROBINSON, Eric. Encouraging children to eat more fruit and vegetables: Health vs. descriptive social norm-based messages. Appetite, v. 100, p. 18-25, 2016.

SMITH, S. L.; CUNNINGHAM-SABO, L. Food choice, plate waste and nutrient intake of elementary- and middle-school students participating in the US National School Lunch Program. Public health nutrition, v. 17, n. 6, p. 1255-1263, jun. 2014.

VIANA, V.; SANTOS, P.; GUIMARÃES, M. J. Comportamento e hábitos alimentares em crianças e jovens: uma revisão da literatura. Psicologia, Saúde e Doenças, v. 9, n. 2, p. 209$231,2008$. 